Ding Die kontinuierliche Druckfiltration Stand der Technik*

\author{
Reinhard Bott, Harald Anlauf \\ und Werner H. StahI**
}

Die kontinuierliche Druckfiltration eignet sich als rein mechanischer und daher verfahrenstechnisch und energetisch vorteilhafter Trennprozeß zur Abscheidung von feinstkornreichen Feststoffsystemen mit hoher Mengenleistung bei $r$ : adriger Restfeuchte.

einem kurzen Abriß werden die physikalischen Grundlagen der Druckfiltration beschrieben. Es folgt ein Apparateüberblick, dann werden die zur Zeit am Markt angebotenen Ausführungen erläutert. Im zweiten Teil dieser Arbeit wird eine Berechnung für die entscheidenden Teilprozesse erläutert.

Bei der Prozeßoptimierung wird gezeigt, wie die gegenüber der herkömmlichen Vakuumfiltration gesteigerte Kuchenbildung und die reduzierte Restfeuchte als Nutzengrößen sowie der erhöhte Gasdurchsatz als Aufwandsgröße die Wirtschaftlichkeit dieses bewährten Trennprozesses in neuer Ausführung bestimmen.
Continuous pressure filtration - new developments. Continuous pressure filtration permitting high throughputs with a low residual moisture content, is a suitable (solely mechanical) means of separating solids systems with high fines contents. It is thus not only technically advantageous but also with regard to energy consumption. The physical fundamentals of pressure filtration are briefly reviewed. A survey of the apparatus follows and the various commercially available models are presented and discussed. Process calculation for the crucial sub-processes of continuous pressure filtration is then explained. The process optimization shows how the advantages of greater cake formation and reduced residual moisture content characterizing the new development and the disadvantage of increased air throughput determine the economy of this successful separation process in comparison to the common vacuum filtration methods.
Die kontinuierliche Druckfiltration ist ein rein mechanischer und somit energiegünstiger Trenn- und Entfeuchtungsprozeß. Das Verfahren eignet sich hauptsächlich zur Trennung von Suspensionen mit hohem Feinstkornanteil, die wegen dieses Anteils bei anderen Verfahren zu erheblichen Filtrationsschwierigkeiten führen. Gegenüber der konventionellen Va$1 \cdots$ umfiltration erlaubt die Druckfiltration im Prinzip ein frei gebbares Filtrationspotential, das entweder durch einen Gasdruck, einen Flüssigkeitsdruck oder einen mechanischen Druck erzeugt wird. Im Rahmen dieser Abhandlung wird über diese drei Wirkmechanismen beim Einsatż in kontinuierlich bzw. in quasikontinuierlich arbeitenden Maschinen berichtet werden. Dabei wird die Filtration mittels eines Gasdifferenzdruckes eindeutig im Vordergrund stehen. Weiterhin beziehen sich die Ausführungen auf die sog. kuchenbildende Filtration, wie sie bei der Verarbeitung von vergleichsweise konzentrierten Suspensionen üblich ist.

\section{Physikalische Grundlagen}

Die wesentlichsten physikalischen Grundlagen der Druckfiltration lassen sich auf eindrucksvolle Weise schnell und ein-

* Vortrag von $R$. Bott auf dem Jahrestreffen der VerfahrensIngenieure, 17. bis 19. Sept. 1986 Straßburg.

** Dr.-Ing. R. Bott, BOKELA Ingenieurgesellschaft für Mechanische Verfahrenstechnik mbH, Haid- und Neu-Str. 10, 7500 Karlsruhe, Dr.-Ing. H. Anlauf und Prof. Dr.-Ing. W. Stahl, Institut für Mechanische Verfahrenstechnik und Mechanik der Universität Karlsruhe (TH), Kaiserstr. 12, 7500 Karlsruhe. fach bei der Diskussion eines klassischen Kapillardruckdiagramms erläutern. Ein solches statisches Kapillardruckdiagramm ist qualitativ in Abb. 1a dargestellt; darin ist der Kapillardruck in einem feinporösen Haufwerk bzw. der zu seiner Überwindung notwendige Differenzdruck als Funktion des Sättigungsgrades aufgetragen.

Der Verlauf der Gleichgewichtskurve ist typisch für einen feinstkornreichen Feststoff, der zu einem feinkapillaren, näherungsweise inkompressiblen Filterkuchen führt.

Das Haufwerk bildet sich während der Kuchenbildungsphase. Durch Anlagerung von mehr oder weniger kompressiblen Feststoffteilchen entsteht ein feinverzweigtes, engporiges Kapillarsystem [1].

Soll nun der gebildete Filterkuchen durch Verdrängung der Porenflüssigkeit entfeuchtet werden, so gilt es, zunächst den Eintrittskapillardruck, der mit der gröbsten Pore korreliert, zu überwinden. Aus Abb. 1a ist zu ersehen, daß bei hohem Eintrittskapillardruck mit der üblichen VakuumfiltrationsDruckdifferenz nur eine vergleichsweise geringe Sättigungsgradabsenkung erzielt werden kann. Wird die Druckdifferenz nur mäßig erhöht, so kann gemäß dem produktcharakteristischen Kurvenverlauf eine bedeutende Verbesserung der Entfeuchtung erzielt werden. Diese Druckdifferenzerhöhung erfordert jedoch die Anwendung von Überdrücken.

Abb. 1b zeigt am Beispiel eines Eisenerzes die Ausführung eines „technischen“ Kapillardruckdiagrammes für die Abhängigkeit $S=\mathrm{f}(\Delta p)$ bei laufender Entfeuchtungszeit $t_{2}$. Bei Absenkung des Sättigungsgrades, z. B. durch Erhöhung des anliegenden Differenzdruckes, steigt der Kapillardruck im Kuchen entlang der Gleichgewichtskurve an. Bei der techni- 


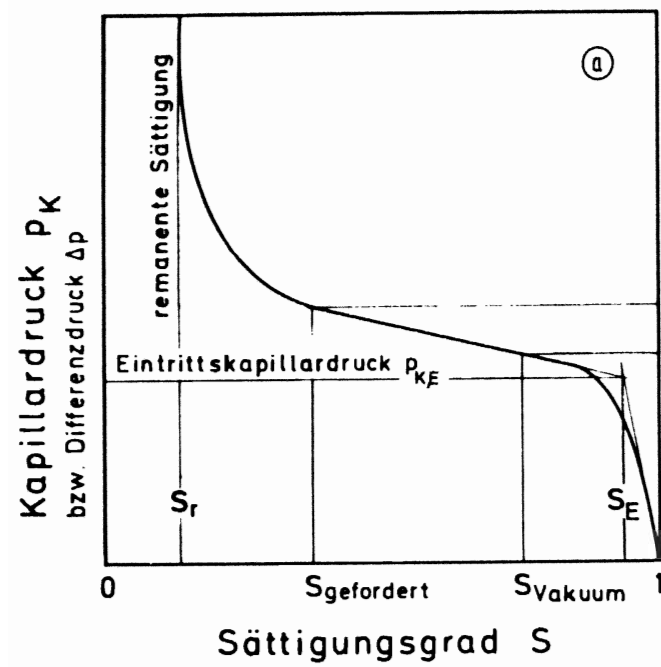

$\Delta p_{\text {erforderlich }}$ $\Delta p=0,8$ bar

$\equiv$ Vakuumfiltrationsdruckdifferenz

$S=\frac{\text { Flüssigkeitsvolumen }}{\text { Hohlraumvolumen }}=\frac{V_{L}}{V_{H}}=-\frac{V_{L}}{\varepsilon \cdot V_{\text {ges }}}$ $(\varepsilon=$ Kuchenporosität)

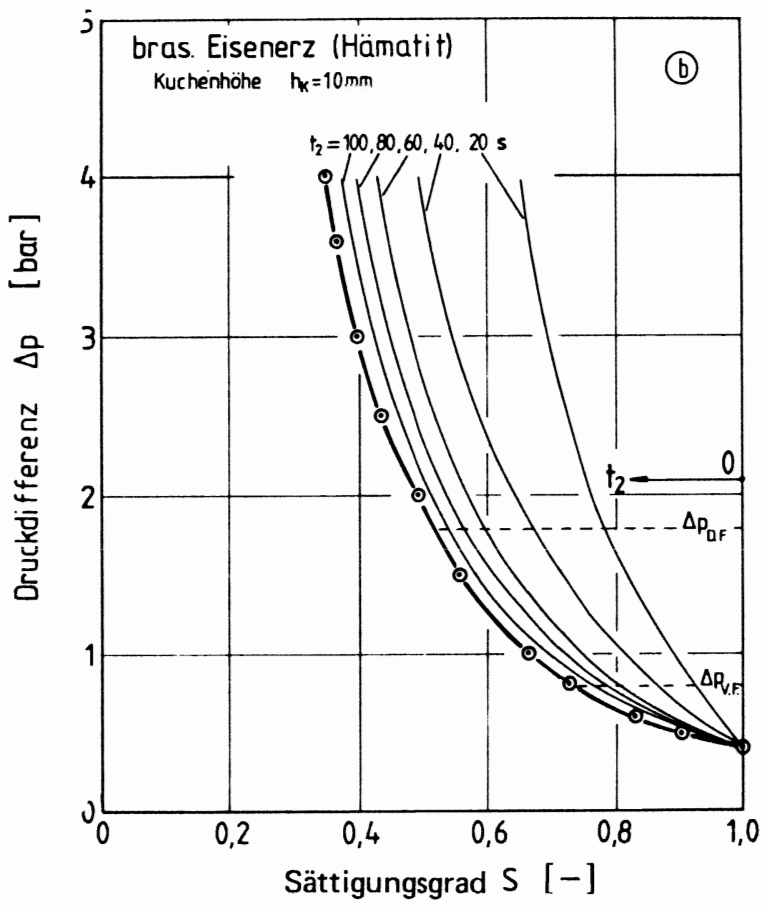

Abb. 1. Kapillardruckkurven.

schen Filtration sind die Entfeuchtungszeiten i. a. zu kurz, als daß sich im Filterkuchen ein Gleichgewichtszustand zwischen $\Delta p, p_{\mathrm{K}}$ und $S$ einstellen könnte. Die Entfeuchtungszeiten variieren häufig im Bereich zwischen 5 und 180 s. In Abb. 1b sind zur Veranschaulichung des zeitabhängigen Entfeuchtungsvorganges die Sättigungsverlaufskurven für vorgegebene Entfeuchtungszeiten $t_{2}$ eingetragen. Für einen geforderten Sättigungsgrad kann mit Hilfe der technischen Entfeuchtungslinien die Betriebseinstellung, bestehend aus Druckdifferenz, Entfeuchtungszeit und Kuchenhöhe, festgelegt werden, die zum gewünschten Ergebnis führt. Unter Verwendung eines solchen technischen Kapillardruckdiagramms ist es möglich, bereits mit geringem Material- und Zeitaufwand weitgehende Aussagen über den absoluten Erfolg und die Effektivität des Druckfiltrationsprozesses zu erstellen.

Das Kapillardruckdiagramm stellt somit eine Charakterisierung, vergleichbar einem Fingerabdruck, eines Filtrationsproduktes dar.

\section{Entwicklung und heutiger Stand der kontinuierlichen Druckfiltration}

Die kuchenbildende Filtration mittels Überdruck wird schon seit vielen Jahrzehnten bearbeitet. Erste technische Ausführungen sind bereits aus dem Jahr 1916 bekannt. Abb. 2 zeigt eine Druckfilteranlage der Fa. Zenith, in der ein konventionelles Trommelfilter arbeitete [2].

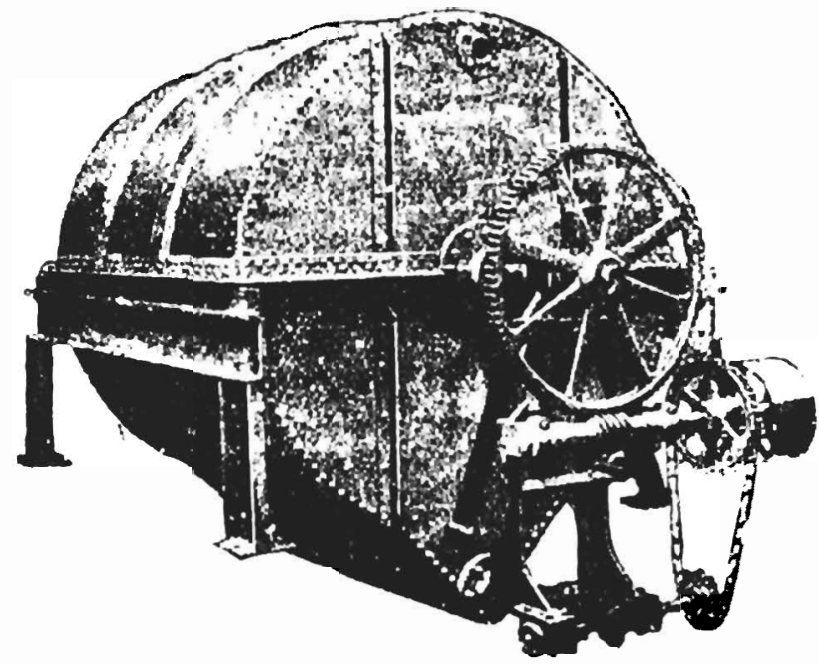

Abb. 2. Kontinuierliches Druck-Trommelfilter aus dem Jahre 1916.

Der neueste Entwicklungsstand auf dem Gebiet der kontinuierlichen Druckfiltration ist gekennzeichnet durch eine Dreiteilung des Apparate- bzw. Wirkprinzips. Wie eingangs bereits erwähnt, kann man in die Rubrik „Kontinuierliche Druckfilter" auch die Apparate einordnen, die den Filterkuchen mittels eines Flüssigkeits- oder eines mechanischen Druckes auf eine geforderte Konsistenz bringen. Zur Abgrenzung des eigentlich angesprochenen Wirkprinzips (Filtration mittels eines Gasdruckes) und zum Aufzeigen der Konkurrenzsituation werden im folgenden zunächst diese beiden Apparatelinien getrennt behandelt.

\subsection{Siebbandpressen}

Das Prinzip der mechanischen Filterkuchenpressung wird vorzugsweise auf Siebbandpressen umgesetzt. Bei diesen kontinuierlich arbeitenden Preßfiltern wird der Schlamm zwischen zwei über Rollen geführten Filterbändern entfeuchtet. In Abb. 3 ist beispielhaft ein Apparateschema dargestellt, das zur allgemeinen Erläuterung des Prozeßablaufs dient. Im ersten Schritt geht es darum, die hochkonzentrierte oder zumeist geflockte Suspension möglichst gleichmäßig auf das Filterband aufzugeben. In der Aufgabezone ist das endlos umlaufende Filtertuch häufig am Rand nach oben gekrempt, um ein Auslaufen der Suspension zu verhindern. In der Seihzone

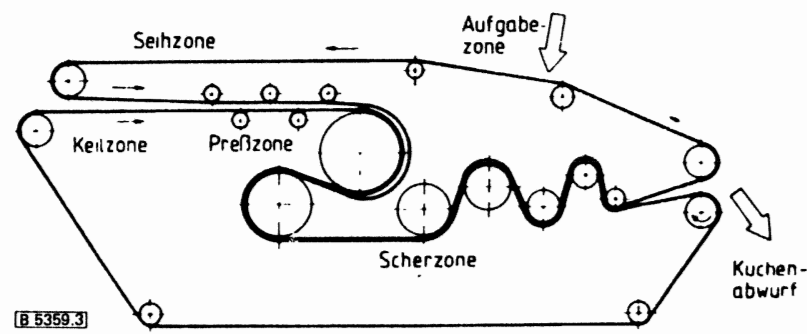

Abb. 3. Apparateschema der Siebbandpresse. 
bildet sich aus dem Schlamm durch Abfluß der freien Flüssigkeit im Erdschwerefeld eine kuchenähnliche Ablagerung, die am Ende der Seihzone formstabil sein sollte. Nach der Seihzone wird das Filterband um eine Umlenkrolle bewegt, der Kuchen bricht $a b$, lagert sich um und wird anschließend in die Keilzone gebracht. Hier beginnt die eigentliche mechanische Kuchenpressung.

Im Gegensatz zu den beiden anderen Druckfiltrationsprizipien beschränkt sich bei der Siebbandpresse die Druckanwendung auf die Entwässerung des Kuchens, während die Kuchenbildung selbst nur unter Wirkung der einfachen Erdschwere abläuft.

Beim Einzug in die Keilzone hat der kompressible Kuchen soweit stabil zu sein, daß er beim Pressen nicht seitlich aus dem Tuch herausläuft. Die Keilzone wird in manchen Fällen auch senkrecht ausgeführt. Durch mehrfaches Umlenken über große und kleine Walzen wird der Kuchen mehrfach gegensinnig geschert, umgelagert und wiederholt ausgepreßt. In der Preßzone können je nach Tuchspannung und Walzenradius Liniendrücke bis $150 \mathrm{~N} / \mathrm{cm}$ und Flächendrücke bis 2 bar realisiert werden. Nach der Preßzone verläßt der hochverdichtete Kuchen den Bandbereich, wobei er allerdings immer noch -ollkommen flüssigkeitsgesättigt ist. Das Band wird anschlieind über Rollen in die Keilzone zurückgeführt, wobei es in dieser Phase gewaschen werden kann [3].

Die Apparategröße liegt heutzutage zwischen 1,0 $\mathrm{m}$ und 3,5 $\mathrm{m}$ Breite. Nach anfänglichem Einsatz in der Umwelttechnik zur Entwässerung von kommunalen und industriellen Abwasserschlämmen hat sich die Siebbandpresse inzwischen einen breiten Markt in der Zellstoff- und Papierindustrie, im Bergbau (z. B. Kohleflotationsberge), in der chemischen Industrie (z. B. Pigmente), in der Erdölindustrie (z. B. Rohölschlamm), in der Lebensmittelindustrie (z. B. Fruchtsaftgewinnung) u. a.m. erobert.

Ungeachtet der mäßigen Kuchenbildung, die durch Einsatz von erheblichen Flockungsmittelmengen teilweise kompensiert wird, bietet diese Art der Druckfiltration durch die mechanische Verdichtung des Haufwerkes bis an die Grenzen der Deformierbarkeit Entwässerungsergebnisse, die sich durchaus mit den Restfeuchtewerten der übrigen Verfahren vergleichen lassen.

\subsection{Preßfilterautomat (Automatische Filterpresse)}

er Preßfilterautomat ist ein Gerät, das die bewährten Eigenschaften der Filterpresse, des Dreh- und Bandfilters in sich vereinigt. Sein Aufbau kombiniert die Konstruktionsmerkmale der Filterpresse mit denen des Bandfilters. Er ist durch die horizontale Anordnung der Filtereinheiten gekennzeichnet, vgl. Prinzipbild Abb. 4 [4].

Der Apparat beruht auf einem russischen Patent. In Europa wird er durch die Firmen Larox, Finnland, und E. Hoesch, Düren, gebaut und vertrieben.

Das Filter arbeitet in einer kontinuierlichen Folge abgeschlossener Zyklen. Ein Arbeitszyklus umfaßt das Schließen, Füllen, Filtrieren, Abpressen mittels Membranen, Waschen, Nachpressen, Trockenblasen, Öfnen, Kuchenaustrag und Tuchreinigung.

Alle diese Vorgänge, von denen der eine oder andere auch übersprungen werden kann, sind individuell programmierbar, so daß der Filtrationsbetrieb in der Regel vollautomatisch abläuft [5].

Dank der kurzen Filterzyklen, deren Dauer zwischen 10 und 30 min. liegt, kann der Preßfilterautomat als quasi-kontinuierlich arbeitendes Gerät eingesetzt werden. Die Gesamttotzeit beträgt einschließlich Kuchenaustrag zwischen 1 und 3 $\min$.

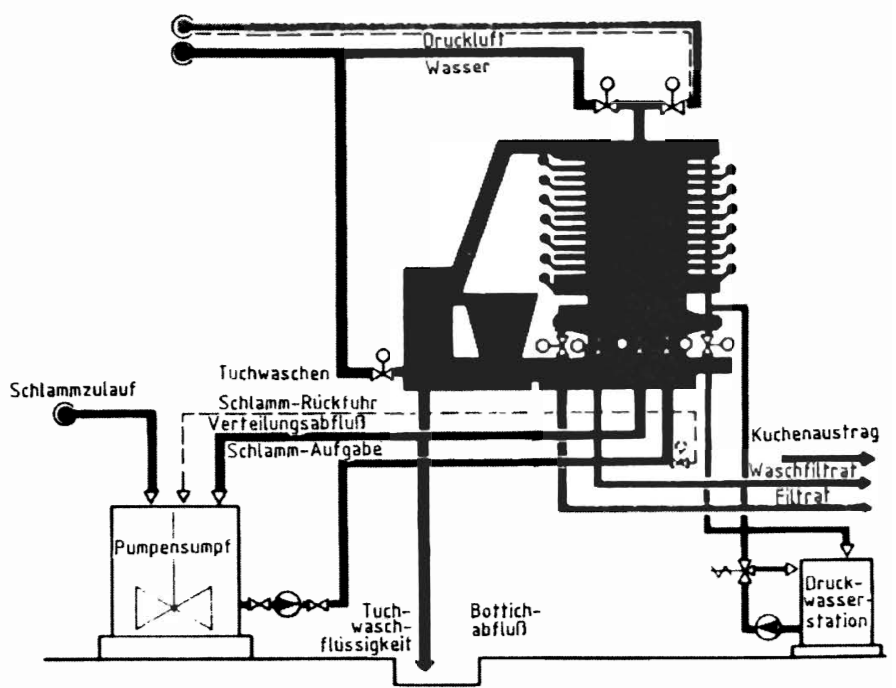

Abb. 4. Apparateschema eines ProzeBfilterautomats (automatische Filterpresse).

Die verfügbare Filterfläche variiert zwischen $1,6 \mathrm{~m}^{2}$ (Hoesch) bzw. 2,5 $\mathrm{m}^{2}$ (Larox) und $32 \mathrm{~m}^{2}$. (Larox) bzw. $36 \mathrm{~m}^{2}$ (Hoesch) bei bis zu 20 Filterplatten. Die installierte Leistung beträgt z. B. bei einer $10 \mathrm{~m}^{2}$-Einheit insgesamt $21 \mathrm{~kW}$ für die Klemmvorrichtung $(7,5 \mathrm{~kW})$, den Tuchspannungsantrieb $(1,1 \mathrm{~kW})$, den Tuchantrieb $(4 \mathrm{~kW})$ und die Hochdruckwasserpumpe (7,5 kW); die Antriebsleistung für ein Luftverdichteraggregat ist im Einzelfall bei der Anlagendimensionierung festzulegen.

Kritische Punkte stellen der Filterrahmen mit den Jochplatten (mechanische Stabilität), das Filtertuch (Endlosverschluß, Verziehen), die Membranen (Druck- und Dauerstandfestigkeit) und die exakte Positionierung des Filterbandes dar. Der nahezu bedienungsfreie Verfahrensablauf hat inzwischen zu einer beachtlichen Verbreitung dieser Technik geführt. Schwerpunktmärkte sind die Chemie- und Nahrungsmittelindustrie (Hoesch) bzw. in hohem Maße die Metallurgie und der Bergbau (Larox). So konnten allein 1986 durch die Firma Larox $611 \mathrm{~m}^{2}$ neu installierte Druckfilterfläche nachgewiesen werden.

\subsection{Drehdruckfilter (Gasdifferenzdruckfilter)}

Diese Kategorie von kontinuierlichen Druckfiltern hat in jüngster Zeit, zum einen bedingt durch die eigene Forschungsarbeit [6], zum anderen bedingt durch neuartige Apparateentwicklungen, den größten Entwicklungsschub erlebt. Gerade im Hinblick auf die bei dieser Filtrationstechnik unumgängliche Ausschleusung des feuchten Schüttgutes „Filterkuchen" wurden wesentliche Fortschritte erzielt.

Das am längsten auf dem Markt befindliche Druckdrehfilter ist das BHS-FEST-Druckfilter. Hierbei handelt es sich um ein Trommelfilter, bei dem sich die Trommel konzentrisch in einem druckfesten Gehäuse dreht, vgl. Abb. 5. Der Ringraum zwischen beiden wird axial durch Stopfbuchsen abgedichtet und am Umfang durch feder- und druckluftbeaufschlagte Trennelemente in druckdichte Kammern unterteilt. Die Zoneneinteilung ist frei wähl- und nachträglich variierbar. Der übrige Filteraufbau ist wie bei einem konventionellen Trommelfilter mit Filterzellen, Filtratrohren und Radialsteuerkopf.

Bei der Universalausführung wird die Suspension unter Druck von unten entgegen der Schwerkraft zugeführt. In den tiefgezogenen Zellen bildet sich ein Kuchen von 5 bis $25 \mathrm{~mm}$ Höhe. In den nachfolgenden Kammern wird der Kuchen je 

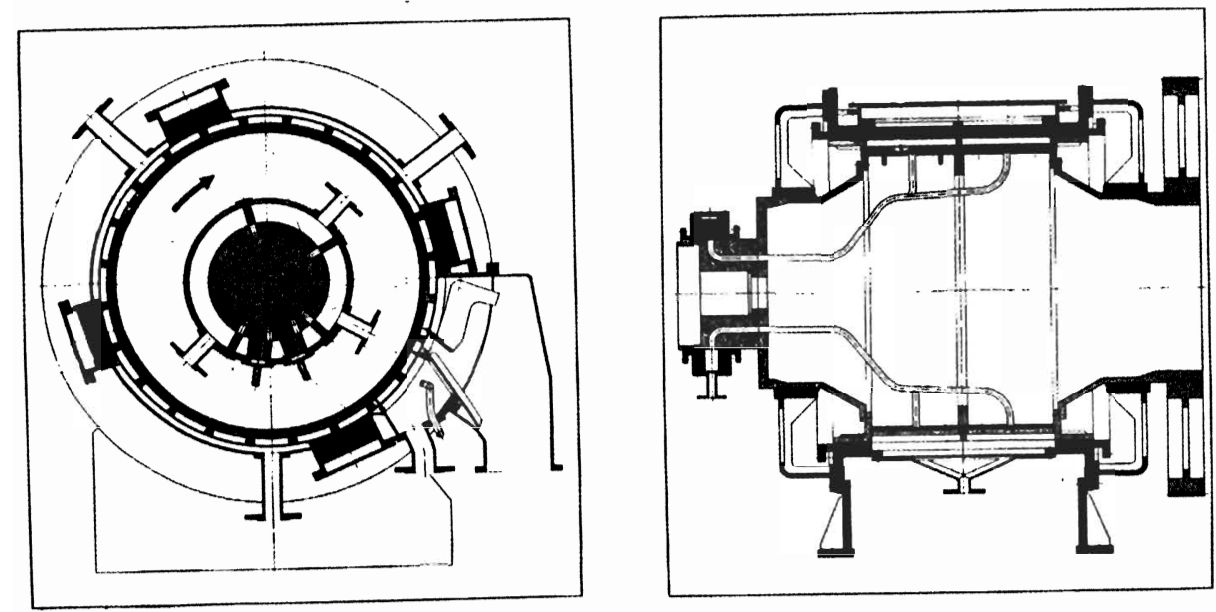

Abb. 5. Apparateschema eines BHSFEST-Druckdrehfilters.

\section{B 5359.5}

nach den Erfordernissen ein- oder mehrstufig gewaschen, extrahiert und gedämpft. Anschließend werden die Kuchenporen mit Druckluft freigeblasen. Die getrennte Aufgabe der Behandlungsmedien in separaten Zonen erlaubt eine ideale

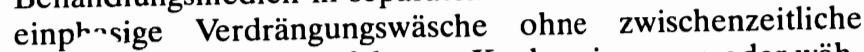
Luft :ührung. Die Gefahr von Kuchenrissen vor oder während der Wäsche ist somit ausgeschlossen.

Nach der Entfeuchtungszone wird der Kuchen in die unter normalem Umgebungsdruck befindliche Kuchenabnahme gebracht. Der Kuchen wird mit einem Schaber, unterstützt durch einen Druckluftrückstoß, aus der Zelle herausgenommen.

Im Anschluß an diese Zone ist eine Vorrichtung zur kontinuierlichen Tuchreinigung vorgesehen.

Das BHS-FEST-Druckfilter wird standardmäßig bis zu $4,3 \mathrm{~m}^{2}$ (in Sonderfällen bis zu 7,7 $\mathrm{m}^{2}$ ) Filterfläche ausgeführt. Der maximale Uberdruck liegt bei 3 bar. Von diesem Filter wurden bisher weltweit ca. 460 Einheiten verkauft.

Aufgrund seines hohen apparatebaulichen Aufwandes und des damit verbundenen hohen Preises hat sich das BHSFEST-Druckfilter nicht zur Verarbeitung von Massengütern durchsetzen können. Vielmehr ist es aufgrund seiner besonderen Eigenschaften häufig auch als "Waschmaschine“ bekannt und wird bevorzugt zur Behandlung hochwertiger Feststoffe unter Luftabschluß eingesetzt. Bei Feststoffen, deren Dichte deutlich von der der Suspensionsflüssigkeit abweicht, bzv ei der Gefahr von Sedimentation, kann die Suspensionszufuhr nach oben verlegt werden.

Entgegen allen anderen Entwicklungen der jüngsten Zeit wurde bei der Fa. AMA-Filter (Alkmaar, NL) ein Druckfiltrationskonzept verfolgt, das auf der vollkommenen Neukonstruktion des Apparates beruht. Das kontinuierliche Druckfilter KDF besteht aus einem horizontalen, zylindrischen Kessel, in dem sechs Filterhohlwellen auf einer gemeinsamen Hauptwelle montiert sind. Jede Filterwelle trägt eine Anzahl (60 bis 120) scheibenförmiger Filterelemente. Die Filterelemente, die beidseitig filtrieren, rotieren ihrerseits auf der Filterwelle planetenartig um die Hauptwelle.

Abb. 6 verdeutlicht schematisch den Filtrationsablauf. Die Kuchenbildung beginnt, wenn eine Filterscheibe voll in der Suspension untergetaucht ist. Das Filtrat fließt durch die Scheibe in die Hohlwelle und über Ventile in einen Abscheider. Sobald die erste Teilfläche aus der Suspension austaucht, wird Druckluft durch die Kuchenporen gedrückt und so die Restfeuchte abgesenkt. Wenn die Filterachse ihren höchsten Punkt erreicht hat, wird das Filtrationsventil geschlossen, und über ein anderes Ventil wird ein Druckluftstoß eingeleitet, der den Filterkuchen vom Metall-Filtertuch abwirft. Der Kuchen fällt in einen Trog, in dem er von einem Kettenförderer zur Austragseinheit transportiert wird. Ǔber die erneute Um- schaltung von Ventilen wird die Kuchenbildung neu gestartet.

Die KDF-Anlage ist serienmäßig in einer Behältergröße lieferbar $(L=9 \mathrm{~m}, B=3,15 \mathrm{~m})$ mit 60,90 und 120 Filterelementen bzw. Quadratmeter Filterfläche. Der maximale Filtrationsdifferenzdruck liegt bei $\Delta p=6$ bar. Das KDF ist gekennzeichnet durch einen ungewöhnlich hohen Stand an Automatisierung und rechnergesteuerter Regelung.

Abb. 7 schließlich gibt ein Bild vom ersten großtechnisch eingesetzten kontinuierlichen AMA-Druckfilter auf der Kohlezeche Zolder in Belgien.
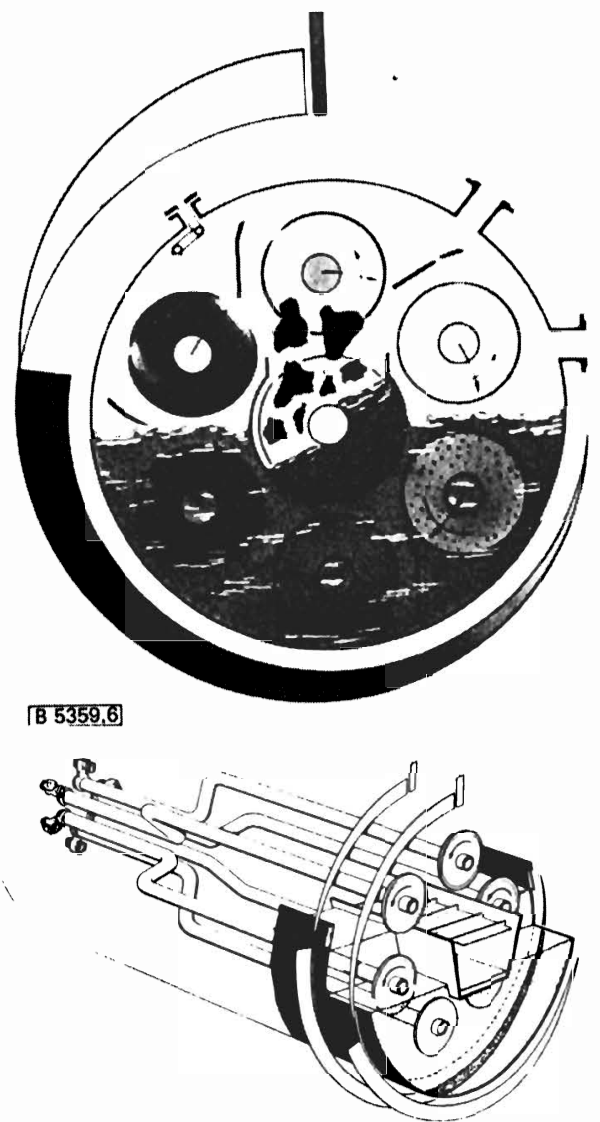

Abb. 6. Schematische Darstellung des Filtrationsablaufes beim kontinuierlichen AMA-Druckfilter.

Besondere Erwähnung verdient an dieser Stelle der Vollständigkeit wegen das AMA-Kuchenaustragssystem. Die Kuchenschleusung erfolgt über ein Ausfallrohr, in dem eine Produktschüttgutsäule als dichtendes Element steht. Die Ku- 


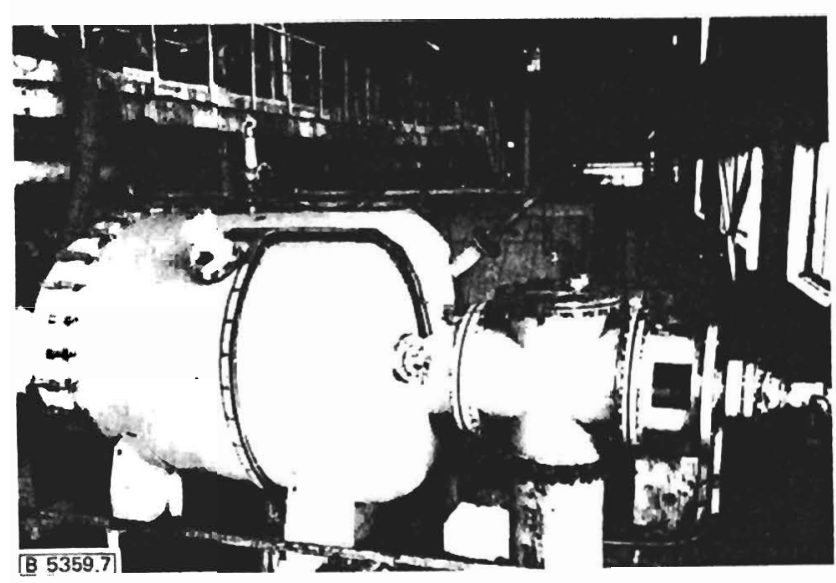

Abb. 7. Kontinuierlicher AMA-Druckfilter auf der Kohlezeche Zolder/Belgien.

chensäule wird unten von einem konischen Verschluß getragen und ist so dimensioniert, daß sicher keine Selbsthemmung entsteht. Beim Austrag wird der Konus kurzzeitig über ein

ydrauliksystem nach unten bewegt. In dieser Phase schießt uer Kuchen unter der Last der Säule und des Überdruckes aus dem Rohr.

\section{Hyperbare (Vakuum)-Filtration}

Die neueste Technik der kontinuierlichen Druckfiltration stellt die im Institut für Mechanische Verfahrenstechnik und Mechanik in Karlsruhe entwickelte Variante dar. Das Verfahren wurde in den vergangenen fünf Jahren bzgl. seiner pyhsikalischen Abhängigkeiten grundlegend untersucht und verfahrenstechnisch bis zur Betriebsreife entwickelt [5].

Das Grundkonzept sieht, wie in Abb. 8 dargestellt, die Installation eines kompletten Drehfilters in einem vertikal geteilten

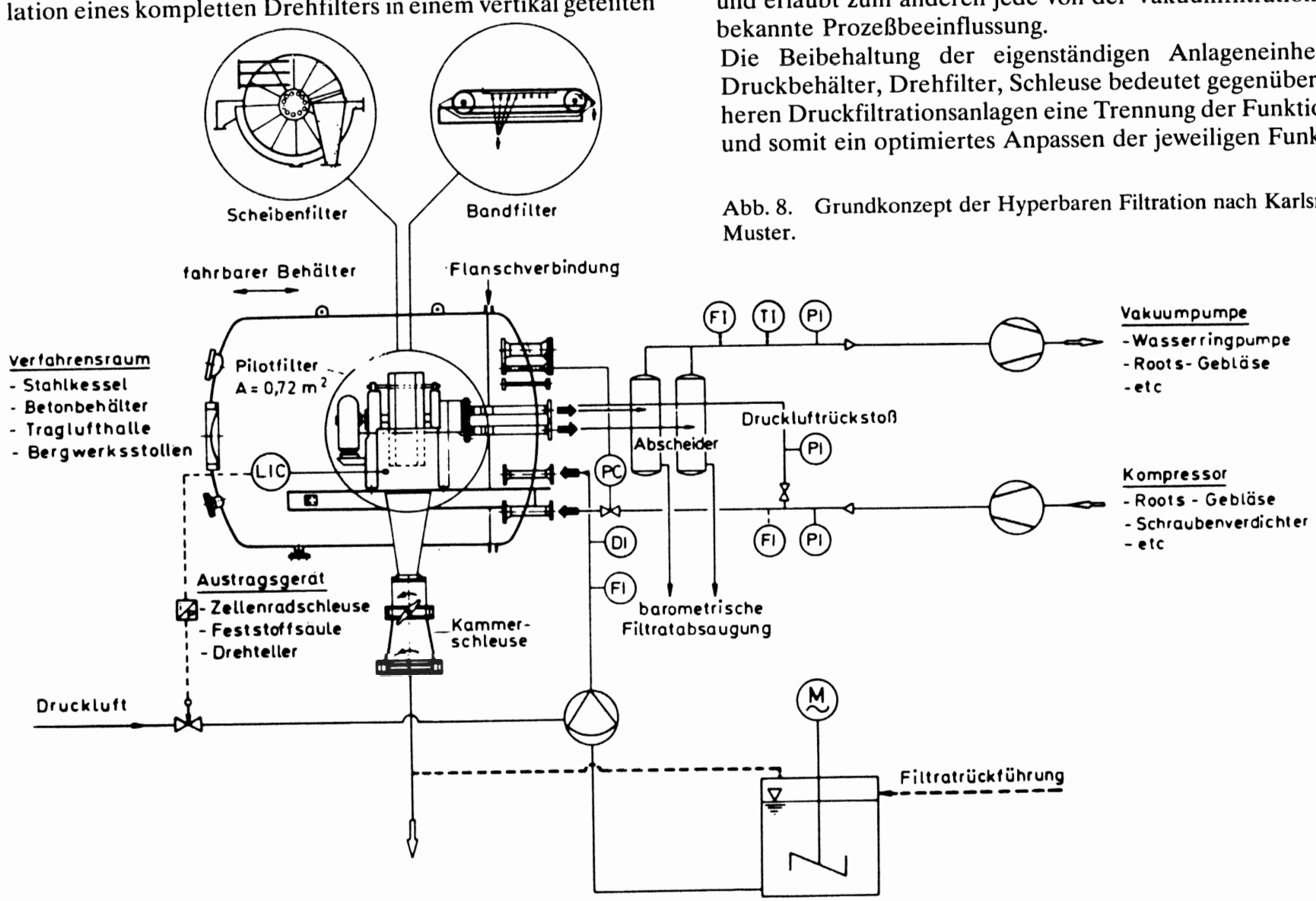

Druckkessel vor. Als Drehfilter bieten sich vorzugsweise alle Typen von Trommel- und Scheibenfiltern an, wobei prinzipiell auch Band- oder andere horizontale Filter in Frage kommen. Die Vollständigkeit der Filtereinhausung schließt sämtliche Filterteile, Antriebe und, entgegen früheren Ausführungen, auch das als Axialsteuerkopf ausgeführte Steuerventil ein.

Der Anlagenbau ist in Anlehnung an bestehende Vakuumfiltrationsanlagen so einfach wie möglich gehalten. Alle Anlagenkomponenten wie konventionelles Drehfilter, Druckbehälter, Suspensionspumpe und Gasverdichter sind so gehalten, daß sie als Standardbauteile eingesetzt werden können [8].

Das Drehfilter ist in dem begehbaren Druckbehälter so angeordnet, daß das Filter von allen Seiten angegangen werden kann, siehe Abb. 9. So können z. B. schnell Scheibensektoren ausgewechselt werden, Filtertuchkontrollen durchgeführt, Antriebe und Getriebe gewartet oder $\mathrm{Zu}$ - und Abläufe verändert werden.

Die Suspension wird von außerhalb über eine geeignete Förderpumpe (Förderdruck $>$ Filtrationsüberdruck + hydrostatischer Suspensionsdruck) in den Filtertrog transportiert. Nach der Fest-Flüssig-Trennung strömt das Filtrat und die Entfeuchtungsluft über die gewöhnlichen Filtratrohre und den Steuerkopf in die Abscheider. Der Filterkuchen fällt nach der Kuchenabnahme auf ein Abzugsband bzw. in den Ausfallschacht, an den die Schleuse angeflanscht ist.

Die Schilderung des Filtrationsablaufs verdeutlicht das wesentlichste Charakteristikum dieser Technik: Im Prinzip handelt es sich um den gewohnten Aufbau einer konventionellen Vakuumdrehfilteranlage, die nun in einem Druckbehälter untergebracht ist. Als ergänzendes Anlagenteil muß dann eine Feststoffschleuse vorgesehen werden.

Die vollkommene Beibehaltung des Vakuumfiltrationsprinzips gewährleistet zum einen eine hohe Betriebssicherheit und erlaubt zum anderen jede von der Vakuumfiltration her bekannte Prozeßbeeinflussung. heren Druckfiltrationsanlagen eine Trennung der Funktionen

Abb. 8. Grundkonzept der Hyperbaren Filtration nach Karlsruher Muster. 

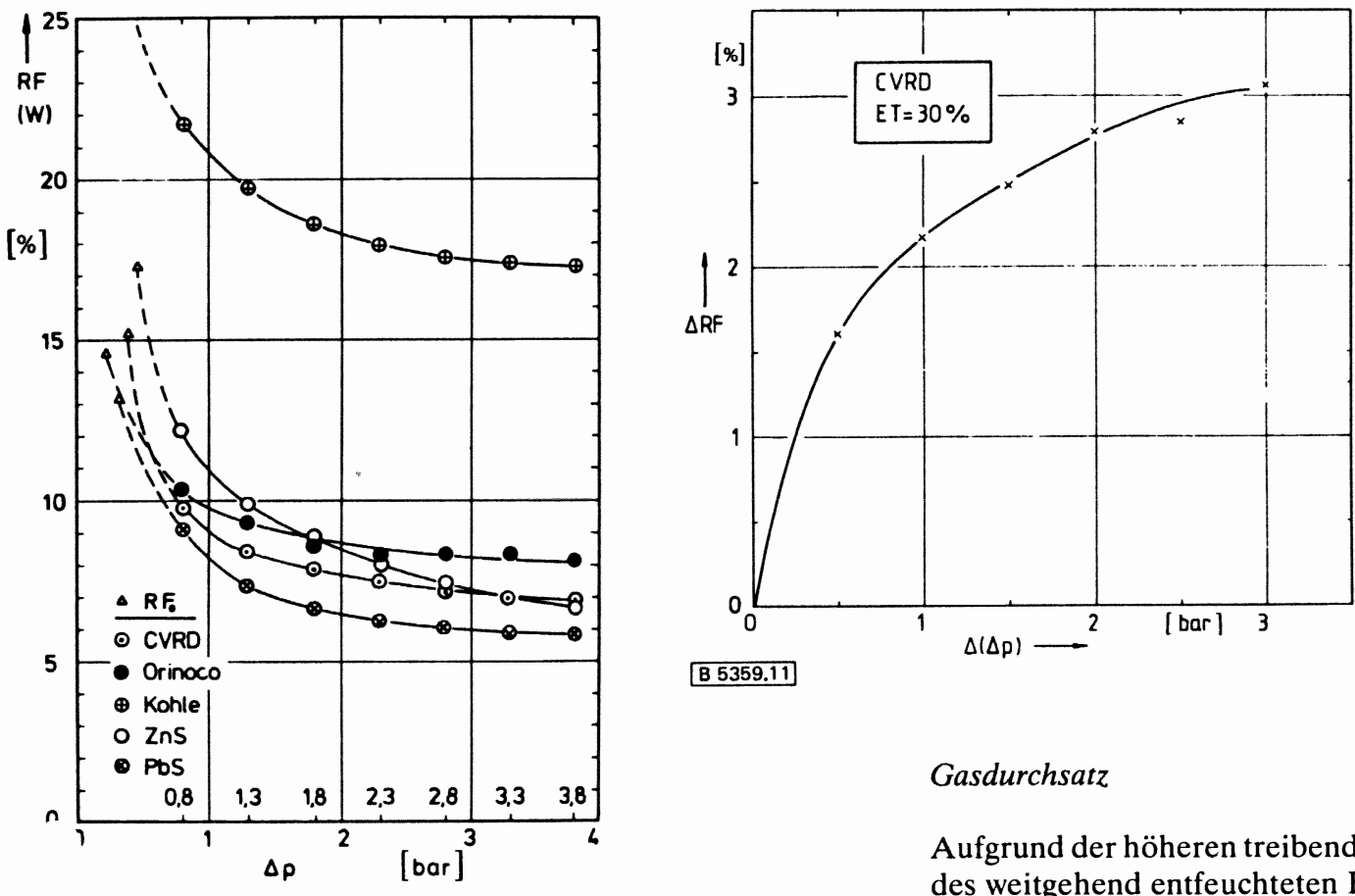

B5359.11
Abb. 11. Restfeuchteverhalten bei der Druckfiltration. den, daß unter Drehfiltrationsbedingungen die Restfeuchteverbesserungsrate gegenüber der reinen Vakuumfiltration mit zunehmender Druckdifferenz schlechter wird.

Darüber hinaus zeigte es sich, daß die Restfeuchte von der Filterdrehzahl und somit von der Kuchenhöhe unabhängig ist, so daß sich i.a. je Druckdifferenz ein Restfeuchtewert ergibt. Weiterhin ist festzustellen, daß die Restfeuchte ausschließlich vom Betrag der Entfeuchtungsdruckdifferenz bestimmt wird und von der Art der Erzeugung (D. F. oder H. V. F.) weitestgehend unabhängig ist.

Zur überblicksmäßigen Vermittlung des Druckfiltrationsertrages empfiehlt sich eine Darstellung nach Abb. 12. Hier ist die Restfeuchte als Funktion des Massendurchsatzes (bei verschiedenen Druckdifferenzen) aufgetragen. Als zusätzlicher Parameter sind die Linien konstanter Filterdrehzahl eingetragen.

\section{Gasdurchsatz}

Aufgrund der höheren treibenden Druckdifferenz und wegen des weitgehend entfeuchteten Filterkuchens nimmt der Gasdurchsatz bei der Druckfiltration gegenüber der Vakuumfiltration gemäß folgender Gl. zu:

$\dot{V}_{\mathrm{g}} \sim \Delta p \frac{p_{\mathrm{m}}}{p_{\mathrm{N}}} \quad, \quad p_{\mathrm{m}}=\frac{p_{\text {über }}+p_{\text {unter }}}{2}$

Gegenüber der Restfeuchte zeigt der Gasdurchsatz eine eindeutige Abhängigkeit von der Filterdrehzahl bzw. der Kuchenhöhe. Dieser Sachverhalt wird in Abb. 13 verdeutlicht, wobei sich in erster Näherung ein linearer Zusammenhang ergibt.

Da sich bei Erhöhung der Druckdifferenz und der Filterdrehzahl auch der Feststoffdurchsatz erhöht, ist für die Anlagendimensionierung bzw. für den Nachweis ihrer Wirtschaftlichkeit letztendlich der durchsatzspezifische Gasdurchsatz

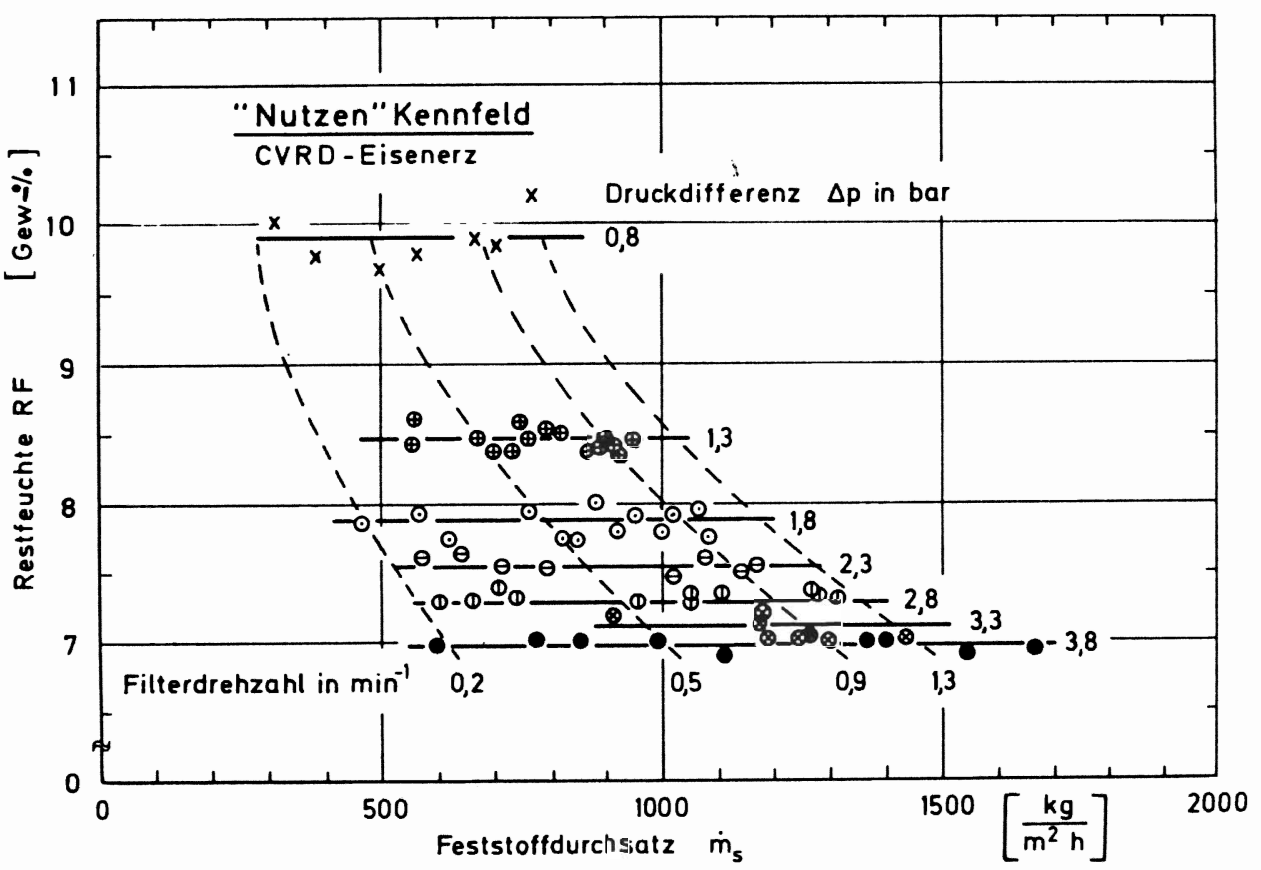

Abb. 12. Ertrags-Diagramm der Hyperbaren Filtration. 

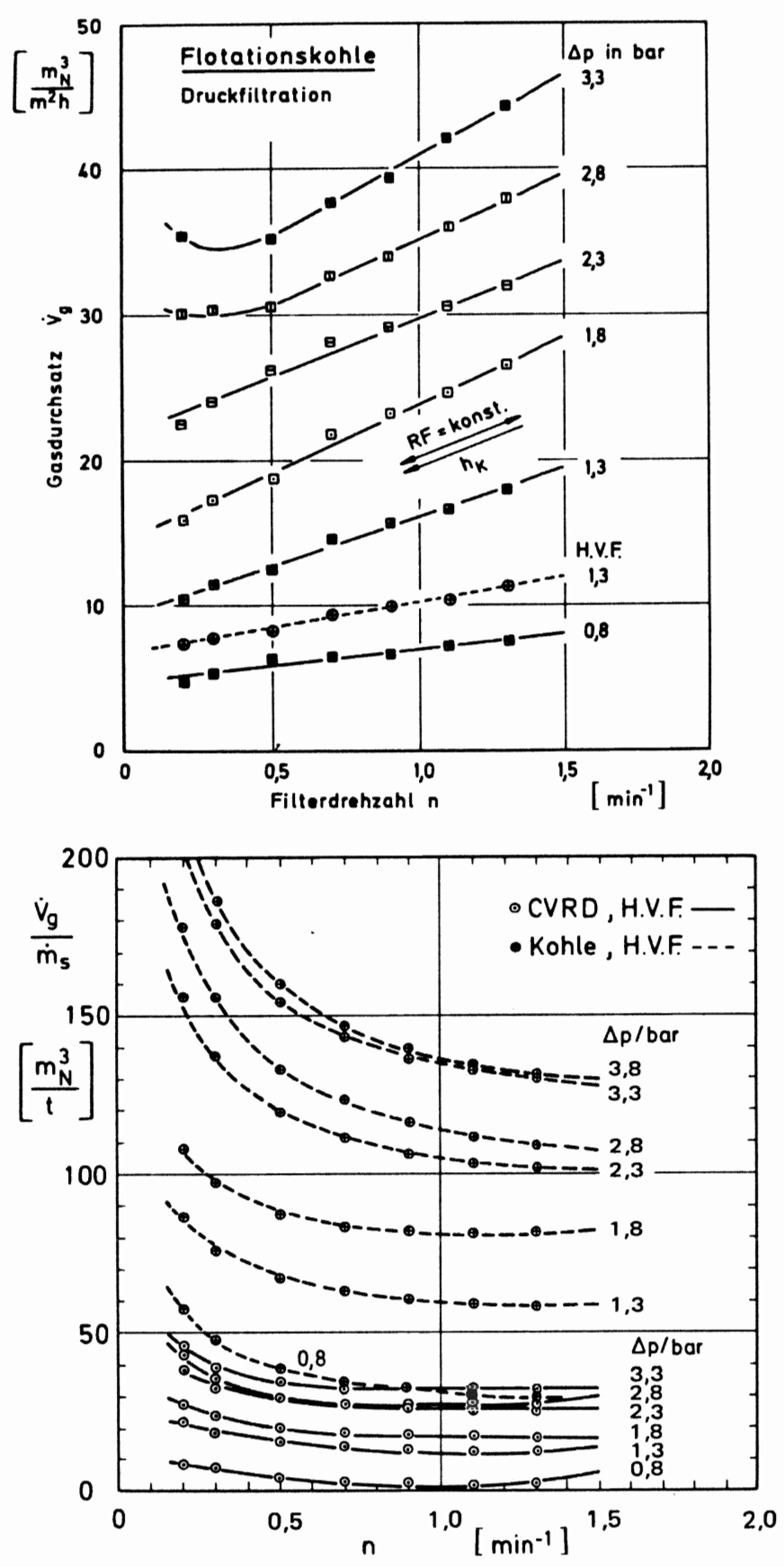

Abb. 13. Gasdurchsatzverhalten bei der Druckfiltration.

entscheidend. Diese Größe $\dot{V}_{\mathrm{g}} / \dot{m}_{\mathrm{s}}$ ist ebenfalls in Abb. 13 als Funktion der Filterdrehzahl aufgetragen.

Der Kurvenverlauf zeigt, daß im Bereich kleiner Durchsätze (und somit kleiner Drehzahlen) die höchsten Aufwandswerte vorliegen. Folglich empfiehlt sich eine Betriebseinstellung im oberen Drehzahlbereich, zumal ab einem bestimmten kritischen Durchsatz der spez. Aufwandswert kaum noch zunimmt. Diese Tendenz zu hohen Drehzahlen deckt sich mit der Forderung nach einem höchstmöglichen Massendurchsatz, der ebenfalls im Bereich hoher Drehzahlen erzielt werden kann.

Bei der weiteren Anlagendimensionierung errechnet sich aus dem Gasdurchsatz zunächst mit Hilfe der Gleichung für die technische Leistung aus der Thermodynamik der theoretische Aufwand für die Verdichtung des Entfeuchtungsluftstromes $(\Delta p=$ const. $)$.

Bei der Bestimmung des Verdichteraggregats und der erfor- derlichen Antriebskapazität ist die derart bestimmte Verdichtungsleistung mit dem Wirkungsgrad des jeweiligen Gerätes zu korrigieren.

In Abb. 14 ist das Blockschaltbild der Dimensionierung einer Druckfiltrationsanlage zusammengestellt. Ausgangspunkt dieser Fallstudie ist eine Vakuumfiltrationsanlage, in der ein Suspensionsstrom $\dot{V}_{\text {SL }}$ filtriert wird. Der anfallende Feststoffstrom $\dot{M}_{\mathrm{s}}$ ist nur ungenügend entfeuchtet und muß folglich nachbehandelt werden. Dazu bietet es sich an, den nassen Filterkuchen thermisch zu trocknen oder mit rückgeführtem trockenem Material oder fremden Zuschlagstoffen zu konditionieren. Beide Methoden sind aufwendig, teuer und mit weiteren Folgeprozessen behaftet (z. B. Staubabscheidung beim Trockner, Befeuerung, Heizstofftankanlagen, etc.). In diesem Fall stellt die kontinuierliche Druckfiltration eine überaus wirtschaftliche Verfahrensalternative dar. Die betriebliche D. F.-Anlage wird derzeit vorzugsweise mit Informationen aus halbtechnischen Pilotversuchen mit einer genügend großen Suspensionsteilmenge dimensioniert.

In einem ersten Schritt wird entsprechend den Prozeßforderungen die Druckdifferenz $\Delta p$ festgelegt, bei der unter Druckfilterbedingùngen die Soll-Restfeuchte $\mathrm{RF}_{\text {Soll }}$ gewährleistet werden kann.

In einem zweiten Schritt wird diejenige Filterdrehzahl $n$ ermittelt, bei der sich beim festgelegten Differenzdruck eine Mindestkuchenhöhe einstellt (ET= const.). Aus der Kuchenhöhe und der Filterdrehzahl ergibt sich der spez. Feststoffdurchsatz $\dot{m}_{\mathrm{s}}$.

Mit dieser Kenntnis wird die zur Bewältigung des Betriebsdurchsatzes $\dot{M}_{\mathrm{s}}$ notwendige Filterfläche $A_{\mathrm{F}}$ festgelegt:

$A_{\mathrm{F}}=\frac{\dot{M}_{\mathrm{s}}}{\dot{m}_{\mathrm{s}}}$

Mit den bisherigen Informationen kann nun das Drehfilter, der Druckbehälter, die Schleuse, die Suspensionspumpe und dgl. festgelegt werden.

In einem dritten Schritt wird der Gasdurchsatz ermittelt, der sich bei der Entfeuchtung des Filterkuchens einstellt. Dieser Grundwert ist zu ergänzen durch das Schöpfvolumen der Filterzellen, die jeweils zu Beginn des Kuchenbildungsvorgangs entleert werden müssen, sowie durch die Steuerkopfleckage und ggf. die Schleusenleckage. Aus dem Gasdurchsatz kann der Kompressor und dessen Antrieb abgeleitet und der wesentliche Teil der Betriebskosten berechnet werden.

Der Versuchsaufwand der Pilotanlage kann durch Vorversuche auf den Laborfiltergeräten „Handfilterplatte“ und „Drucknutsche“ drastisch reduziert werden. Hierzu genügt bereits ein kleines Suspensionsmuster.

\section{Optimierung der Betriebseinstellung}

Der beschriebene Weg der Anlagendimensionierung baut auf vorgegebenen Betriebsgrößen wie Soll-Restfeuchte, Steuerkopfwinkel, Eintauchtiefe, Soll-Kuchenhöhe etc. auf. Vielfach wird damit jedoch nicht die optimale Betriebseinstellung getroffen. Darüber hinaus ist eine Möglichkeit zur Abschätzung der Betriebskosten bei weiterer Restfeuchteabsenkung wünschenswert.

Für diese.Aufgabe wurde die Methode der Entfeuchtungskennfelder geschaffen, wie dies in Abb. 15 qualitativ dargestellt ist [1].

In einem 3-dimensionalen Diagramm ist zunächst die Restfeuchte in Abhängigkeit der Kuchenhöhe und das Entfeuchtungsdifferenzdruckes aufgetragen. Für eine bestimmte Entfeuchtungszeit $t_{2}=$ const. ergibt sich eine im Raum stehende Fläche. Für längere Entfeuchtungszeiten sinkt die Restfeuch- 


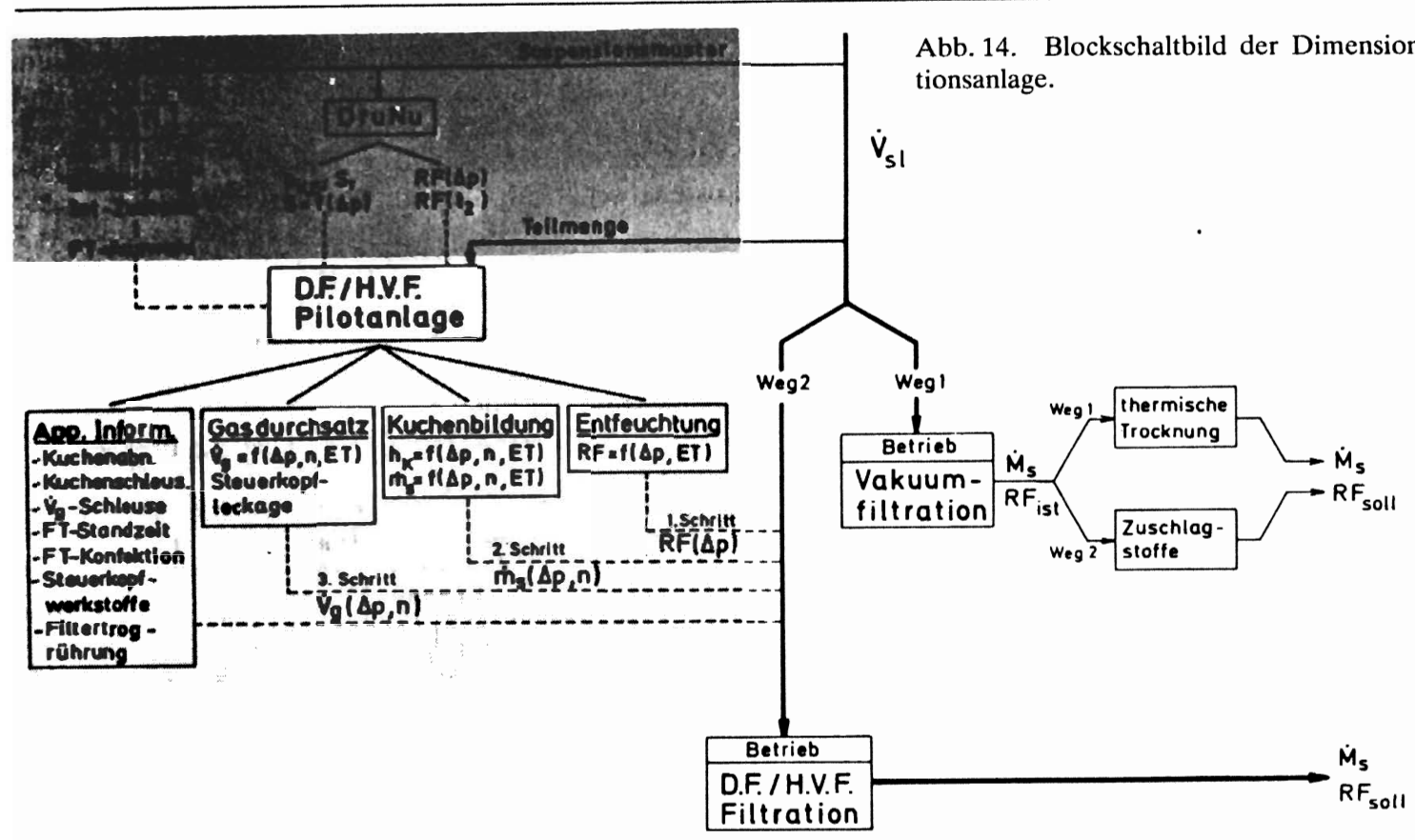

te und somit die Raumfläche stetig ab. Die Eckpunkte der Raumfläche werden durch Laborversuche bestimmt. Für die Verbindungslinien bestehen Interpolationsprogramme. Diese Vorgehensweise garantiert eine größtmögliche Produktnähe.

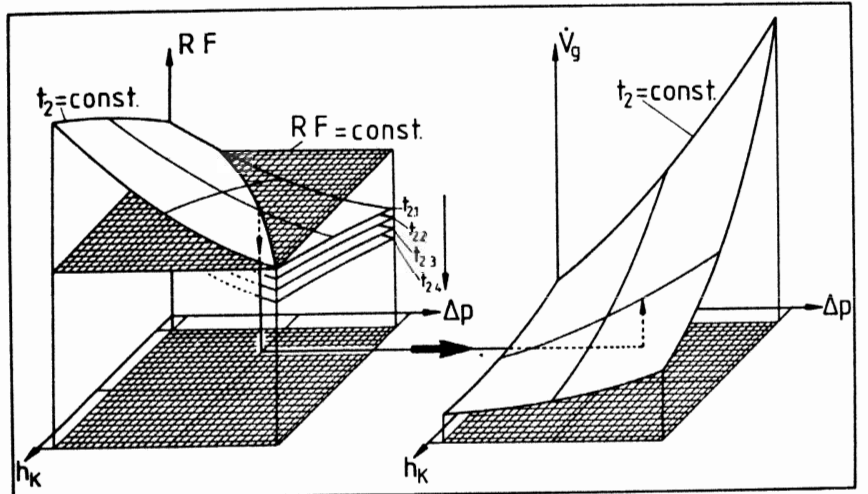

b. 15. Entfeuchtungskennfeld der Druckfiltration.

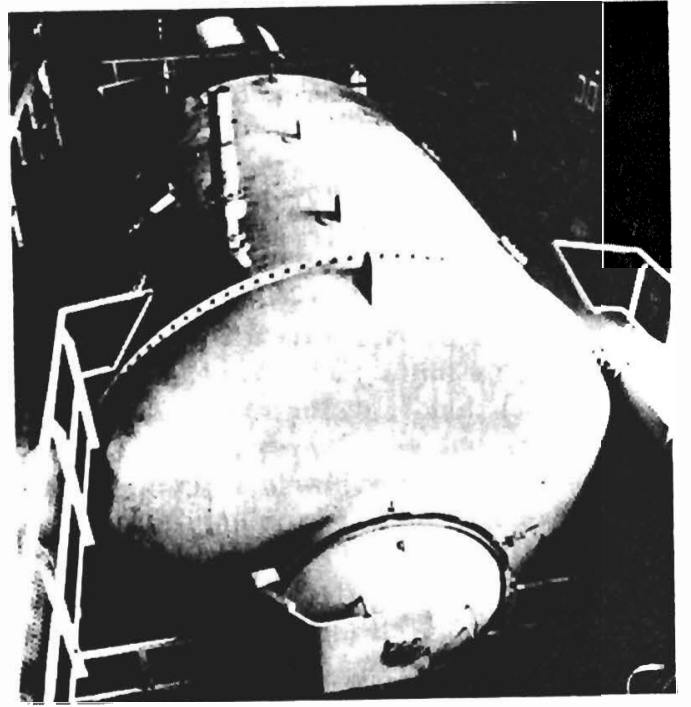

Abb. 16. Industrielle Ausführungen der kontinuierlichen Druckfiltration nach Karlsruher Muster von KHD.
Für eine angenommene Restfeuchte $\mathrm{RF}=$ const. resultiert eine horizontale Ebene. Die Verschneidungslinie der Flächen $\mathrm{RF}=$ const. $/ t_{2}=$ const. liefert sämtliche Kombinationen der Kuchenhöhe $h_{\mathrm{K}}$ und der Druckdifferenz $\Delta p$, bei denen diese Forderungen erfüllt werden.

In einem zweiten 3-dimensionalen Diagramm ist dagegen der Gasdurchsatz ebenfalls in Abhängigkeit der Kuchenhöhe $h_{\mathrm{K}}$ und der Druckdifferenz $\Delta p$ aufgetragen. Die Úbertragung einer zuvor festgelegten Betriebseinstellung in dieses Aufwands-Diagramm liefert nun Angaben über den damit verknüpften Gasdurchsatz. Schließlich gelangt man durch Umrechnung dieser Grundwerte auf die Drehfiltrationsbedingungen zu den für die jeweilige Betriebseinstellung gültigen Betriebskosten.

Mittels einer computergestützten Fassung dieses schrittweisen Optimierungsverfahrens ist es möglich, verschiedene Betriebseinstellungen zu simulieren und den betriebswirtschaftlich günstigsten Fall aufzudecken.

\section{Schlußbemerkungen}

Nach der ausführlichen Erörterung der Verfahrenstechnik und der Anlagengestaltung stellt sich am Ende die Frage: Für welche Produkte ist diese bekannte Technik in neuartiger Ausführung gedacht? Wo liegen die zukünftigen Märkte? Und weiterhin: Wer kann diese Technik liefern? Und last but not least: Was kostet die erforderliche Apparatur?

Die meisten bisherigen halbtechnischen Untersuchungen sowie die ersten großtechnischen Anwendungen liegen im Bereich der Filtration von Aufbereitungstrüben wie Eisenerz-, Zink-, Kupfer-, Flußspat-, Schwerspat- und Kohle-Trüben. Weitere Ansatzpunkte ergaben sich z. B. in der Lebensmittelindustrie im Zuge der Stärke- und Zuckergewinnung oder in der chemischen Industrie (z. B. Farbstoffe). Bei allen Produkten handelt es sich um Massengüter aus Naßverfahren. Die Partikelgrößenverteilungen dieser Feststoffe tendieren eindeutig zum Feinen hin; in jedem Fall lag die mittlere Partikelgröße $x_{50,3}<60 \mu \mathrm{m}$, vielfach gar $x_{50,3}<30 \mu \mathrm{m}$. In neuerer Zeit ist es durch Einsatz neuartiger Filtergewebe gelungen, Feststoffe mit $100 \%<20 \mu \mathrm{m}$ bzw. mittleren Partikeldurchmessern $x_{50,3}<5 \mu \mathrm{m}$ abzuscheiden (z. B.: feinstkörniger Schwerspat, Stärkederivate). 


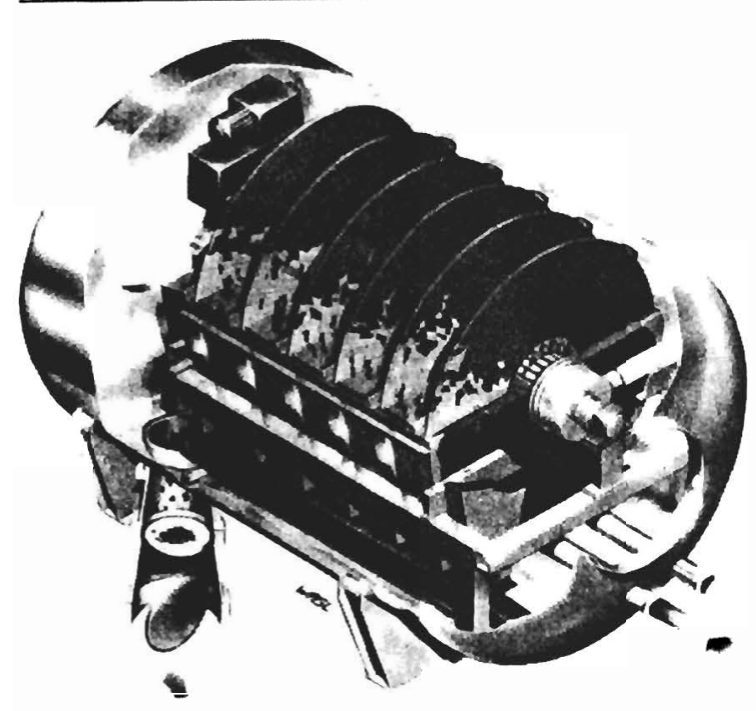

Abb. 17. Industrielle Ausführungen der kontinuierlichen Druckfiltration nach Karlsruher Muster von Andritz.

In jedem Fall führt diese Art der kontinuierlichen Druckfiltration zu einem leicht bröckeligen, feinstückigen Filterkuchen, der ohne nochmals zusätzlich gebrochen werden zu müssen, problemlos transportiert, gelagert, gefördert oder resuspendiert werden kann.

Die Größenordnung von kontinuierlich arbeitenden Druckfiltrationsanlagen wird, von Extremfällen abgesehen, im Bereich von ( 5 bis) 10 bis $100 \mathrm{~m}^{2}$ Filterfläche, entsprechend 20 bis $400 \mathrm{~m}^{2}$ Vakuumfilterfläche liegen.

Die bisher größte Druckfiltrationsanlage nach dem Karlsruher Muster wurde auf der Schachtanlage Emil-Mayrisch des Eschweiler Bergwerkvereins für Flotationskohle gebaut. Die Filterfläche beträgt dort $A_{\mathrm{F}}=96 \mathrm{~m}^{2}$ auf der Basis von Scheibenfilter von KHD [10]. Im Bereich der Kohlefiltration ist die KHD Filterbau, Bochum, in der Lage, komplette Druckfilteranlagen zu liefern.

Desweiteren bietet die Lurgi GmbH, Frankfurt/M., im Zuge ihrer internationalen Mining-Aktivitäten die kontinuierliche Druckfiltration an.

In neuerer Zeit hat die Maschinenfabrik Andritz, Graz, die ntinuierliche Druck- und hyperbare Vakuumfiltration in „ur Fest/Flüssig-Trennprogramm aufgenommen. Dadurch wurde eine markterweiternde Ergänzung zu der neuen Scheiben- und Trommelfiltergeneration erreicht. In Anlehnung an ihre breitgefächerten Erfahrungen mit den Andritz-Siebbandpressen führt dieser Hersteller ein umfassendes Marktangebot bzgl. der kontinuierlichen Druckfiltration.

Bei den Kosten pro Quadratmeter Druckfilterfläche ist gegenüber dem Vakuumfilter der zusätzliche apparative Auf- wand für den druckdichten Betrieb des konventionellen Drehfilters zu berücksichtigen. Hauptkostenfaktoren sind dabei neben dem Filter selbst der Druckbehälter und nicht zuletzt die Filterkuchenschleuse. Als Anhaltswert für eine Investitionskostenabschätzung darf angenommen werden:

Vakuumfiltration

(Scheiben-/Trommel-Filter)

3000 bis $5000 \mathrm{DM} / \mathrm{m}^{2}$ Filterfläche

Druckfiltration

(Scheiben-/Trommel-Filter)

10000 bis $15000 \mathrm{DM} / \mathrm{m}^{2}$ Filterfläche

automatisierte Filterpresse ca. $30000 \mathrm{DM} / \mathrm{m}^{2}$ Filterfläche Scheibenfilter KDF $56000 \mathrm{DM} / \mathrm{m}^{2}$ Filterfläche

Hierbei ist zu berücksichtigen, daß bei der quasikontinuierlichen automatisierten Filterpresse eine ca. 4 bis 5 fach größere Filterfläche pro Feststoffdurchsatz erforderlich ist.

Bei der weiteren Markteinführung dieser Filtertechnik bestehen noch eine große Anzahl effizienter Entwicklungsmöglichkeiten, wie z. B. bei der Erprobung weiterer Schleussysteme oder der Vermeidung von Rißbildung im Filterkuchen [11]. In Zukunft wird mehr und mehr daran gedacht werden, die Kompressionswärme, die bei der Verdichtung der Blasluft automatisch entsteht, dadurch auszunutzen, daß der Druckraum mit heißer Luft beaufschlagt wird.

Eingegangen am 23. Februar 1987 [B 5359]

\section{Literatur}

[1] Anlauf, $H .:$ Dissertation, Univ. Karlsruhe; Fortschr. Ber. VDI Reihe 3, Nr. 144, VDI-Verlag Düsseldorf 1986.

[2] Dickey, G. D.: Filtration; Reinhold Publishing Corp., New York, Chapman \& Hall Ltd., London 1961.

[3] Zoidl, H.: Aufbereit. Tech. (1979) Nr. 4.

[4] Weidner, H.: Zuckerindustrie 103 (1978) S. 311/313.

[5] Haarti, I.: Aufbereit. Tech. (1981) Nr. 5, S. 247/253.

[6] Stahl, W.; Bott, R.; Anlauf, $H .:$ Abschlußbericht zum AIFForschungsvorhaben Nr. 5564, Karlsruhe 1985.

[7] Bott, R.: Dissertation, Univ. Karlsruhe 1986; Heft 49 der Schriftenreihe der GDMB, ISSN 0720-1877.

[8] Bott, R.; Stahl, W.: Symposium Solid/Liquid Separation practice and the Influence of New Techniques, University of Leeds, Apr. 1984.

[9] Persönliche Informationen der Firma Dosiertechnik Schmittinger, 8901 Gessertshausen, Am Mühlanger 7.

[10] Thelen, H.; Wesp, M.; Wüster, G.: Aufbereit. Tech. (1986) Nr. 5, S. $262 / 269$.

[11] Anlauf, H.; Bott, R.; Stahl, W.; Krebber, A.: Aufbereit. Tech. (1985) Nr. 4, S. 188/196. 